\title{
Dynamic correlation diagrams for sigmatropic reactions based on orbital phase conservation theory
}

\author{
Hiroyuki Nohira ${ }^{*} \dot{+}$ and Toshiyuki Nohira ${ }^{\dagger}, \S$ \\ *Department of Applied Chemistry \\ Faculty of Engineering, Saitama University \\ Shimo-okubo 255, Sakura-ku, Saitama 338-8570, Japan \\ $\dagger$ Institute of Advanced Energy \\ Kyoto University, Uji, Kyoto 611-0011, Japan \\ th.nohira@jcome.home.ne.jp \\ $\S_{\text {t-nohira@iae.kyoto-u.ac.jp }}$
}

Received 2 April 2017

Accepted 21 July 2017

Published 8 August 2017

\begin{abstract}
For sigmatropic reactions, such as $[1,3]$ and $[1,5]$ hydrogen shifts, the Cope and Claisen rearrangements, and the Berson-Nelson rearrangement, no correlation diagrams consistent with the classical electronic formulas have been reported. Here, we report dynamic correlation diagrams for these sigmatropic reactions based on orbital phase conservation theory. The diagrams are consistent with both the selection rules of sigmatropic reactions proposed by Woodward and Hoffmann and classical electronic formulas.
\end{abstract}

Keywords: Bond theory; dynamic correlation diagram; orbital phase conservation theory; sigmatropic rearrangement.

\section{Introduction}

The conservation of orbital symmetry theory presented by Woodward and Hoffmann ${ }^{1}$ is one of the most significant theories in the history of organic chemistry. Correlation diagrams play an important role in this theory, and Neumann-Wigner's noncrossing rule ${ }^{2}$ has been almost universally applied when drawing such diagrams. However, very few correlation diagrams can be drawn reasonably if one observes the noncrossing rule, ${ }^{3,4}$ which is based on the assumption that the change of the electronic state is adiabatically slow. ${ }^{2}$ Hence, the noncrossing rule should not be applied to cases in which the change of the electronic state is fast. ${ }^{4}$ In fact, Neumann and Wigner indicated that the crossing of energy levels is possible when the change is

\$Corresponding author.

This is an Open Access article published by World Scientific Publishing Company. It is distributed under the terms of the Creative Commons Attribution 4.0 (CC-BY) License. Further distribution of this work is permitted, provided the original work is properly cited. 


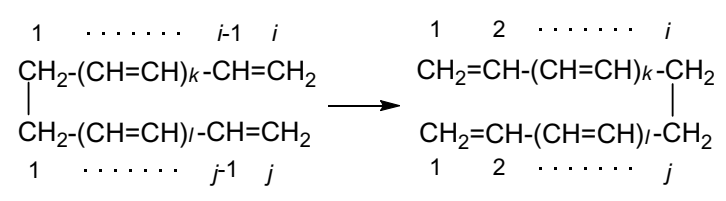

(1)

(2)

Scheme 1. $[i, j]$ Sigmatropic reaction.

fast. ${ }^{2}$ Considering that elementary reactions have been shown to proceed in the $10^{-13}$ to $10^{-14} \mathrm{~s}$ time frame, ${ }^{5}$ the application of the noncrossing rule is no longer necessary in drawing electronic state correlation diagrams. ${ }^{4}$

Based on this observation, we propose an orbital phase conservation theory using dynamic correlation diagrams ${ }^{4}$ that consistently unify Fukui's frontier orbital (FO) theory ${ }^{6}$ and Woodward-Hoffmann's orbital symmetry conservation $(\mathrm{W}-\mathrm{H})$ theory. ${ }^{1}$ In our previous paper, ${ }^{4}$ we reported dynamic correlation diagrams for the DielsAlder reaction, interconversions between hexatriene and cyclohexadiene, and electrophilic substitution of naphthalene, which are representative examples of cycloadditions, electrocyclic reactions, and reactions explained by the FO theory, respectively.

Another important group of reactions explained by the $\mathrm{W}-\mathrm{H}$ theory are sigmatropic rearrangements. ${ }^{7}$ Woodward and Hoffmann defined a sigmatropic change of order $[i, j]$ as the migration of a $s$ bond, flanked by one or more $p$ electron systems, to a new position whose termini are $i-1$ and $j-1$ atoms removed from the original bonded loci, in an intramolecular process (Scheme 1). ${ }^{7}$

A generalized selection rule is typically used to determine whether such a reaction is allowed in either the ground or excited state, and correlation diagrams are generally not thought to be relevant for the analysis of these reactions because the reactants or products may not possess symmetry elements. ${ }^{7}$ Thus, no correlation diagrams for sigmatropic reactions that are consistent with the classical electronic formulas have been reported so far. Appropriate correlation diagrams can be drawn, however, according to the dynamic correlation diagram method we have proposed. In the present paper, we present five new correlation diagrams for sigmatropic reactions: a [1,3] hydrogen shift of propene, a [1,5] hydrogen shift of 1,3-pentadiene, the Cope and Claisen rearrangements, and the Berson-Nelson rearrangement.

\section{Methods}

The dynamic correlation diagrams have been drawn in the same style used in a previous paper. ${ }^{4}$ The approximate energy levels of the orbitals of the reactants are written from top to bottom on the left-hand side of each diagram; those of the products are given on the right-hand side. The simple Hückel method ${ }^{8}$ is generally sufficient for the calculation of energy levels and has been used in this study. Since the bonding energies of a $\mathrm{C}-\mathrm{C}$ single bond and a $\mathrm{C}=\mathrm{C}$ double bond are 83 and $147 \mathrm{kcal} \mathrm{mol}^{-1}$, respectively, the bonding energy for the $\pi$ bond of the double bond 


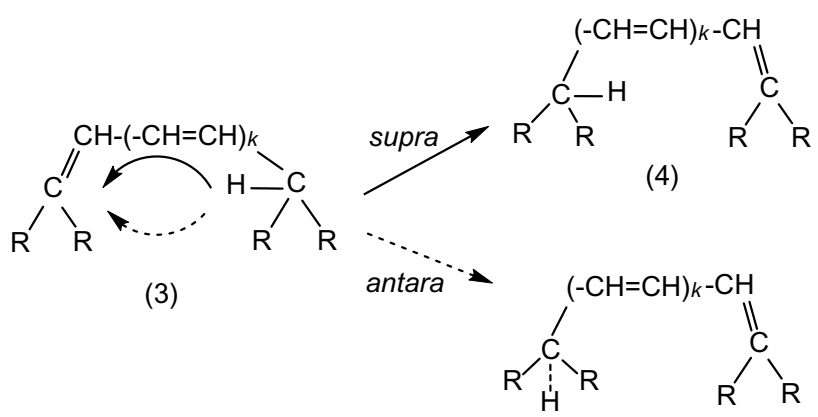

(5)

Scheme 2. $[1, j]$ Sigmatropic reaction.

should be approximately $64(147-83) \mathrm{kcal} \mathrm{mol}^{-1}$. This value corresponds to the bonding energy of the overlap integral $\beta$ obtained by the Hückel method. Thus, the energy levels of a $\mathrm{C}-\mathrm{C} \sigma$ bond and a $\mathrm{C}-\mathrm{H} \sigma$ bond are defined as $1.3(83 \div 64) \beta$ and $1.5(99 \div 64) \beta$, respectively. Next, we consider the change of the key orbitals, i.e. where bond cleavage occurs in the reactant and where the bond formation occurs in the product. Then, we can easily draw a correlation line by considering the changes in the form and energy level of the molecular orbital. Finally, the remaining orbitals can be linked by applying the principle of minimum change of orbital phases. ${ }^{4}$ The reactions of $c i s$-type polyenes in which a hydrogen atom on the $j$ position migrates to position 1 is called a $[1, j]$ sigmatropic reaction (Scheme 2).

\section{Results and Discussion}

When the transferred hydrogen atom is associated with the same face of the $p$ system, the shift is defined as suprafacial. When the migrating hydrogen atom is passed from one face of a carbon terminus to the opposite face of the other, it is defined as antarafacial. The isomerizations of propene and 1,3-pentadiene are [1,3] and $[1,5]$ sigmatropic reactions, respectively.

Figures 1 and 2 show the dynamic correlation diagrams of sigmatropic $[1,3]$ and $[1,5]$ hydrogen shifts of propene and 1,3-pentadiene, respectively.

We can easily derive the selection rules for these reactions using the correlation diagrams shown in Figs. 1 and 2. In the suprafacial process of Fig. 1, the C-H $\sigma$ bonding orbital — the key orbital of the reactant - is linked to the $\mathrm{C}-\mathrm{H} \sigma^{*}$ antibonding orbital of the product. Thus, this reaction is forbidden in the ground state. In contrast, the $\mathrm{C}-\mathrm{H} \sigma^{*}$ antibonding orbital of the reactant is linked with the $\mathrm{C}-\mathrm{H} \sigma$ bonding orbital of the product. Hence, this reaction is allowed in the excited state.

In the antarafacial process of Fig. 1, the $\mathrm{C}-\mathrm{H} \sigma$ bonding orbital of the reactant is linked to the $\mathrm{C}-\mathrm{H} \sigma$ bonding orbital of the product. Therefore, this reaction is allowed in the ground state. In contrast, the $\mathrm{C}-\mathrm{H} \sigma^{*}$ antibonding orbital of the 
H. Nohira \& T. Nohira

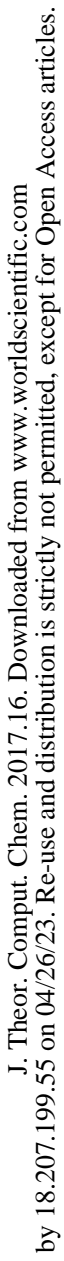

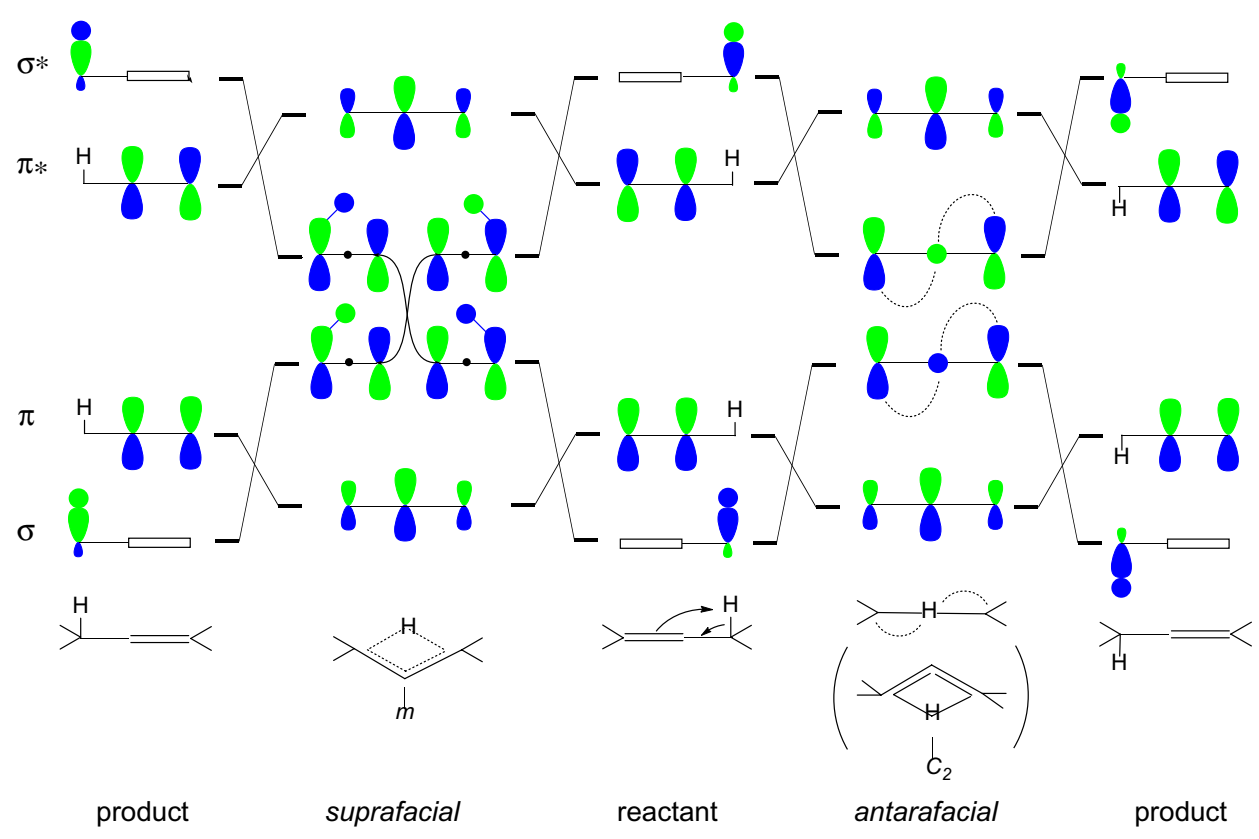

Fig. 1. Dynamic correlation diagram for the sigmatropic $[1,3]$ hydrogen shift of propene.

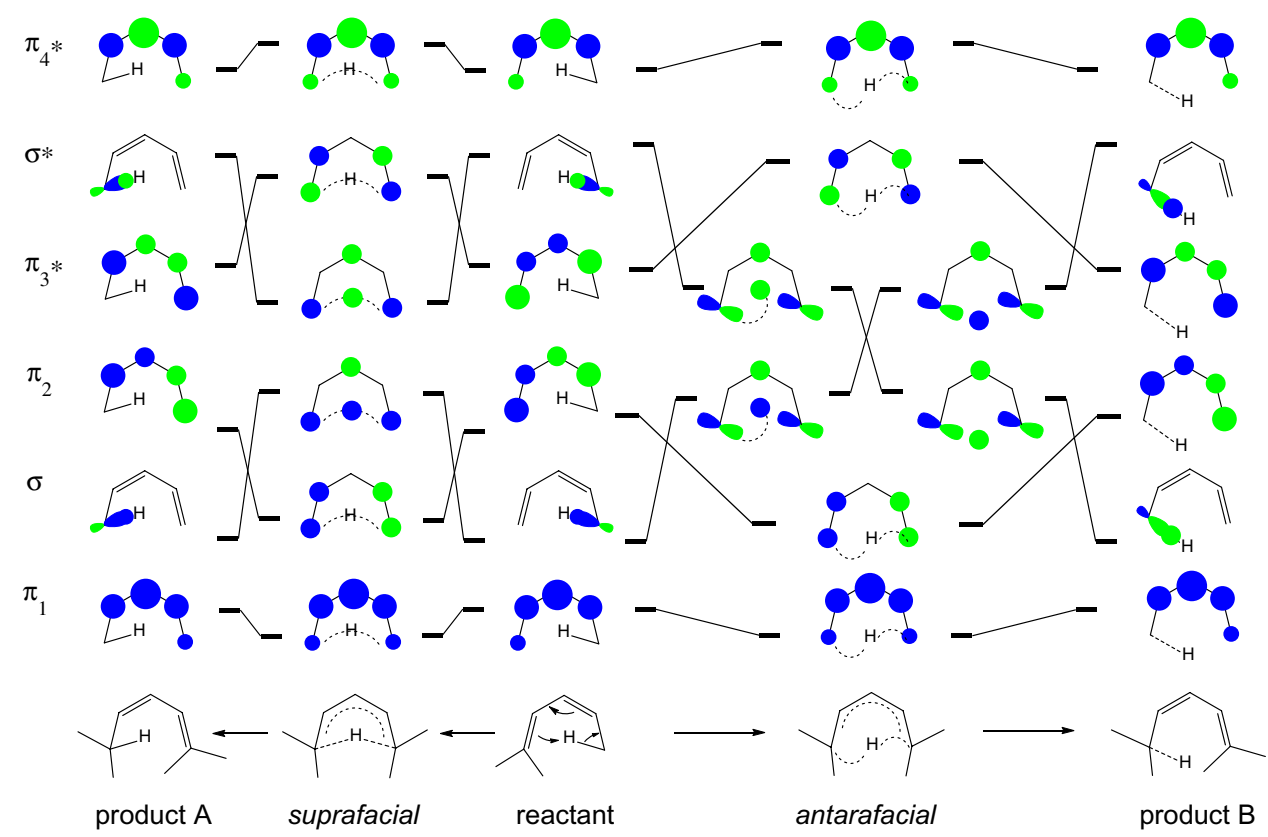

Fig. 2. Dynamic correlation diagram for the sigmatropic $[1,5]$ hydrogen shift of 1,3-pentadiene. 
Table 1. Selection rules for sigmatropic hydrogen shifts of polyenes.

\begin{tabular}{lcc}
\hline & Ground state & Excited state \\
\hline$[1,3]$ suprafacial & Forbidden & Allowed \\
{$[1,3]$ antarafacial } & Allowed & Forbidden \\
{$[1,5]$ suprafacial } & Allowed & Forbidden \\
{$[1,5]$ antarafacial } & Forbidden & Allowed \\
\hline
\end{tabular}

reactant is not linked to the $\mathrm{C}-\mathrm{H} \sigma$ bonding orbital of the product. Thus, this reaction is forbidden in the excited state. For both suprafacial and antarafacial processes, the movement of electrons within the molecule are consistent with classical electronic formulas, as shown in the schemes of Fig. 1.

Similarly, the selection rules for the [1,5] hydrogen shift of 1,3-pentadiene are shown in Fig. 2. The movement of electrons in the molecule agrees with the classical electronic formulas for both the suprafacial and antarafacial processes. Table 1 summarizes the derived selection rules, which are identical to those of sigmatropic reactions generalized by Woodward and Hoffmann. ${ }^{9}$

The Cope rearrangement ${ }^{10}$ is a $[3,3]$ sigmatropic reaction in which the $\mathrm{C}-\mathrm{C} \sigma$ bond at the 3,4-position in 1,5-hexadiene migrates to the 1,6-position to form a new $\sigma$ bond (Scheme 3). Similarly, the Claisen rearrangement ${ }^{11}$ is a $[3,3]$ sigmatropic reaction followed by a $[1,3]$ rearrangement (Scheme 4 ).

The [3,3] sigmatropic shift in 1,5-hexadienes has been shown to proceed more easily through a chair-like transition state than through the boat-like alternative. ${ }^{12}$ Assuming that the C2 symmetry of the axis between the carbon atoms of the 3,4position remains, the correlation diagram is as shown in Fig. 3. According to the diagram shown in Fig. 3, this rearrangement is thermally allowed in the ground state. Moreover, the diagram is consistent with classical electronic formula, as indicated in the scheme of Fig. 3 .

If there exists some steric restriction, this rearrangement can also proceed through the boat-like transition state. Assuming that the mirror symmetry $(m)$ that bisects the $\mathrm{C}-\mathrm{C}$ bond of the 3,4-position remains, the correlation diagram is drawn as shown in Fig. 4. Again, this rearrangement is shown to be thermally allowed in the ground

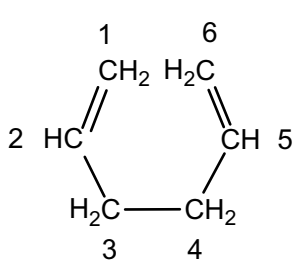

(6)

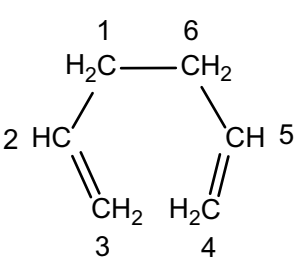

(7)

Scheme 3. Cope rearrangement. 


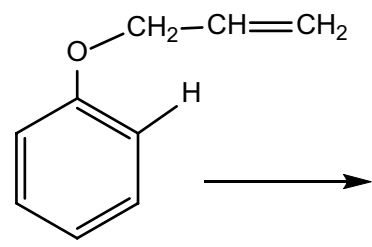<smiles>C=CCC1C=CC=CC1=O</smiles>

(8)

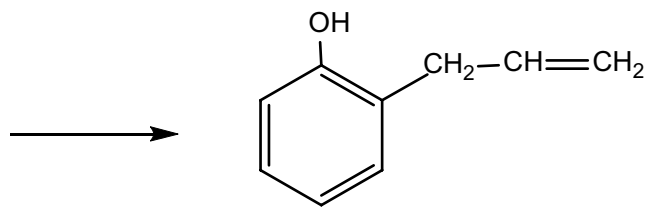

(9)

Scheme 4. Claisen rearrangement.

state and the diagram is compatible with the classical electronic formula shown in the scheme of Fig. 4.

Woodward and Hoffmann also developed a correlation diagram for this reaction through the boat-like transition state, to which the noncrossing rule was applied. ${ }^{13}$ Their correlation lines are shown in Fig. 4 as dotted lines. According to this diagram, the movement of electrons in the molecule is expressed by the classical electronic formula of Scheme 5, which is unrealistic. This example demonstrates why the noncrossing rule is not generally suitable for correlation diagrams.

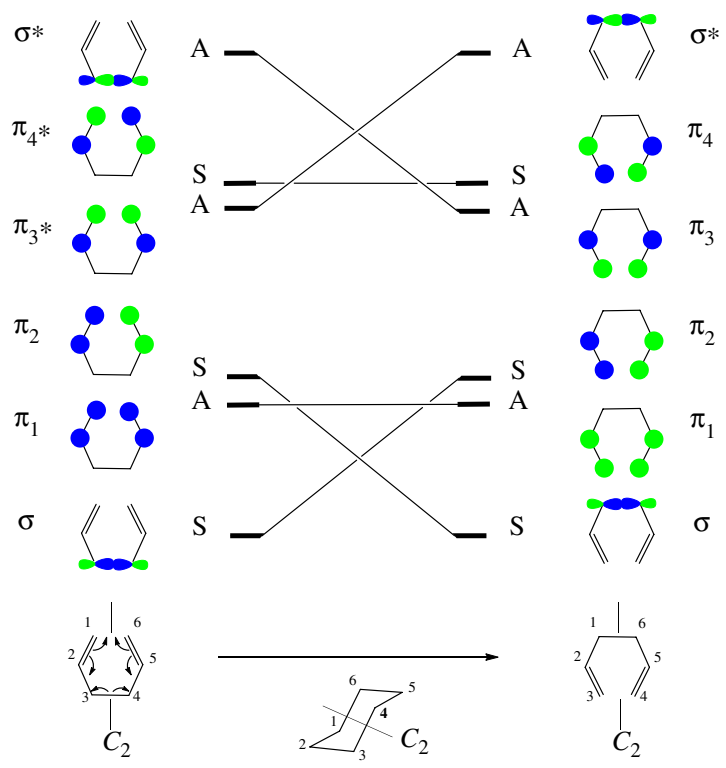

Fig. 3. Dynamic correlation diagram for the Cope sigmatropic $[3,3]$ rearrangement of 1,5 -hexadiene via the chair conformation. 


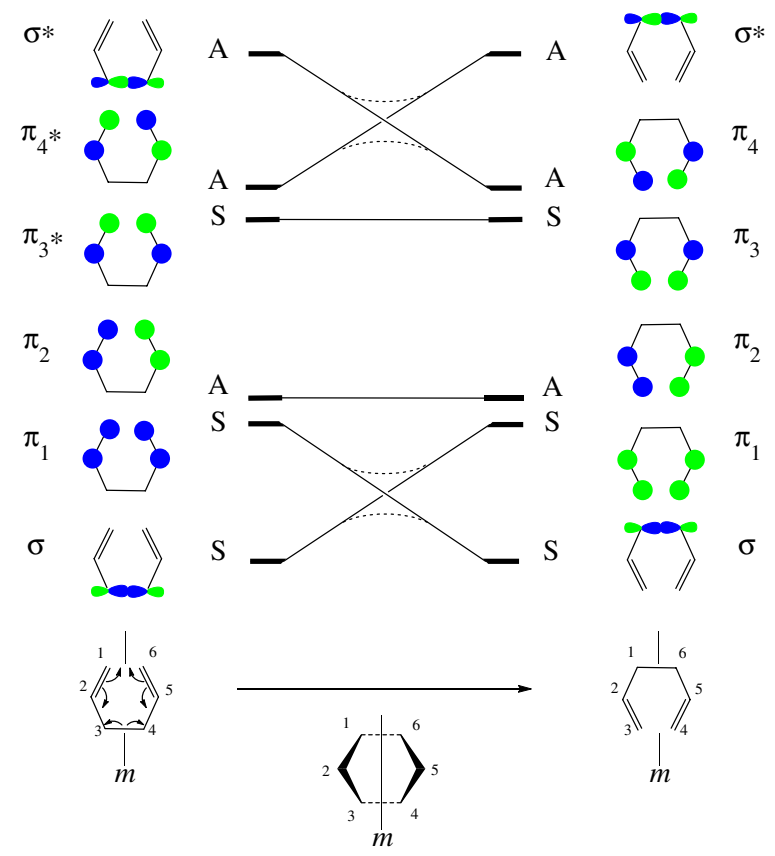

Fig. 4. Dynamic correlation diagram for the Cope sigmatropic [3,3] rearrangement of 1,5-hexadiene via the boat conformation.

As shown in Fig. 1, [1,3] suprafacial hydrogen shifts are known to be thermally forbidden. However, for cases in which the shifting atom is not hydrogen and the new bond is formed by inversion of the orbital configuration of the migrating carbon atom, the reaction becomes thermally allowed. Woodward and Hoffmann give the Berson-Nelson rearrangement as an example, ${ }^{14}$ but do not include a correlation diagram. Since there is no symmetry maintained during this reaction, the appropriate correlation diagram cannot be drawn if the noncrossing rule is to be followed.

However, ignoring the noncrossing rule and incorporating the minimum change of orbital phases, ${ }^{4}$ an appropriate correlation diagram (Fig. 5) can be drawn using the dynamic correlation method. The movement of electrons in the molecule is consistent with classical electronic formula, as indicated in the scheme of Fig. 5.

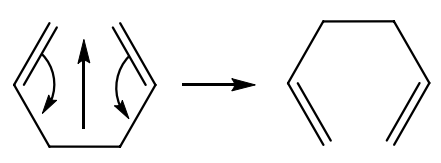

Scheme 5. Movement of electrons corresponding to the correlation diagram drawn by Woodward and Hoffmann (dotted lines of Fig. 4). 

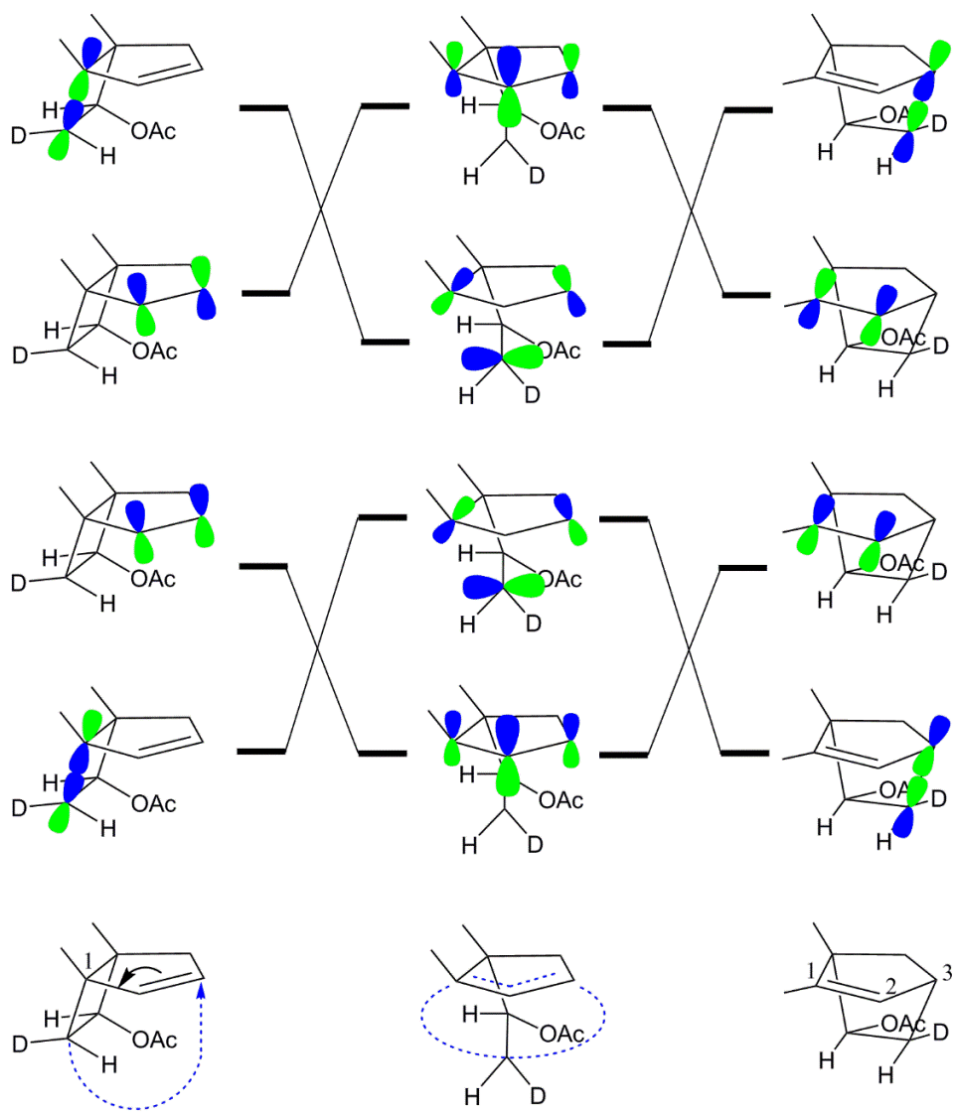

Fig. 5. Dynamic correlation diagram for the Berson-Nelson sigmatropic $[1,3]$ rearrangement.

\section{Conclusion}

Based on orbital phase conservation theory, we have successfully drawn dynamic correlation diagrams for selected sigmatropic reactions, none of which has heretofore been deemed appropriate for such diagrams. Since the newly drawn correlation diagrams are completely consistent with the classical electronic formulas, they may become a definitive theoretical basis for the empirically well-established $\mathrm{W}-\mathrm{H}$ rules.

\section{Acknowledgment}

T. N. thanks Ms. H. Kadowaki for the help in drawing dynamic correlation diagrams.

\section{References}

1. Woodward RB, Hoffmann R, The conservation of orbital symmetry, Angew Chem Int Ed 8:781-853, 1969; Woodward RB, Hoffmann R, The Conservation of Orbital Symmetry, 1st ed., Verlag Chemie, GmbH, Weinheim, 1970. 
2. von Neuman J, Wigner E, On the behavior of eigenvalues in adiabatic processes, Z. Physik 30:467-470, 1929.

3. Nohira H, Correlation diagrams for the concerted $\pi-\sigma$ bond transformation reactions, Tetrahedron Lett 30:2573-2576, 1974.

4. Nohira H, Nohira T, Quantization of chemical reaction: The dynamic correlation diagram method free from noncrossing rule, J Theor Comput Chem 11:379-389, 2012.

5. Zewail AH, Recent progress in studies of dynamics and control of reactions and their transition states, J Phys Chem 100:12701-12724, 1996.

6. Fukui K, Yonezawa T, Shingu H, A molecular orbital theory of reactivity in aromatic hydrocarbons, J Chem Phys 20:722-725, 1952; Fukui K, Recognition of stereochemical paths by orbital interaction, Acc Chem Res 4:57-64, 1971.

7. Woodward RB, Hoffmann R, Selection rules for sigmatropic reactions, J Am Chem Soc 87:2511-2513, 1965; Woodward RB, Hoffmann R, The Conservation of Orbital Symmetry, 1st ed., Verlag Chemie, GmbH, Weinheim, pp. 114-151, 1970.

8. Streitwieser A Jr, Molecular Orbital Theory for Organic Chemists, John Wiley \& Sons, Inc., New York, pp. 33-62, 1961.

9. Woodward RB, Hoffmann R, The Conservation of Orbital Symmetry, 1st ed., Verlag Chemie, GmbH, Weinheim, p. 117, 1970.

10. Cope AC, Hardy EM, The introduction of substituted vinyl group. V. A rearrangement involving the migration of allyl group in a three-carbon system, J Am Chem Soc 62:441444, 1940; Levy H, Cope AC, The rearrangement of allyl groups in three-carbon systems. IV. Hydrocarbons, J Am Chem Soc 66:1684-1688, 1944.

11. Claisen L, Über die Umlagerung von phenylallyl äthern in die isomer allylphenole, Justus Liebigs Ann Chem 418:69-120, 1919.

12. von E. Doering W, Roth WR, Thermal rearrangements, Angew Chem Int Ed 2:115-122, 1963; Hill RK, Gilman NW, Asymmetric induction in the Cope rearrangement, Chem Commun 619-620, 1967; Simonetta M, Favini G, Mariani C, Gramaccioni P, Calculated energies and geometries along the reaction path in Cope rearrangements, J Am Chem Soc 90:1280-1289, 1968.

13. Woodward RB, Hoffmann R, The Conservation of Orbital Symmetry, 1st ed., Verlag Chemie, GmbH, Weinheim, p. 148, 1970.

14. Berson JA, Nelson GL, Inversion of configuration in the migrating group of a thermal 1,3sigmatropic rearrangement, J Am Chem Soc 89:5503-5504, 1967; Berson JA, The stereochemistry of sigmatropic rearrangements. Test of the predictive power of orbital symmetry rules, Accounts Chem Res 1:152-160, 1968. 